\title{
Study of lighting solutions in machine vision applications for automated assembly operations
}

\author{
Alberto Zorcolo* , Gustavo Escobar-Palafox, Rosemary Gault, Robin Scott and \\ Keith Ridgway
}

AMRC, University of Sheffield, Advanced Manufacturing Park, Wallis Way, Catcliffe, Rotherham, S60 5TZ, UK

*corresponding author: $\underline{\text { a.zorcolo@amrc.co.uk }}$

\begin{abstract}
The application of machine vision techniques represents an invaluable aid in many fields of manufacturing, from part inspection to metrology, robot guidance and assembly operations in general. An effective illumination of the working area constitutes a crucial aspect for optimising the performance of such techniques but unfortunately ideal light conditions are rarely available, especially if the vision system has to work within small areas, possibly close to metallic surfaces with high reflectivity. This work aims to investigate which factors mostly affect the accuracy in a typical feature recognition and measurement application. A first screening of a set of six factors was carried out by testing three different light sources, according to a two-level fractional factorial design of experiments (DOE), a Pareto analysis was performed in order to establish which parameters were the most significant. Once the key factors were identified, a second series of the experiments were carried out on a single light source, in order to optimise the key parameters and to provide useful guidelines on how to minimise measurement errors in different scenarios.
\end{abstract}

\section{Introduction}

Traditional automation in assembly is often associated to serial production with large batch sizes and limited flexibility [1]. This means that usually a single automated manufacturing line only suits a particular type or a small family of similar products, and introducing new components would involve significant changes in the assembly line, with obvious repercussion on costs and production time. Also complexity in certain assembly processes may be an obstacle when it comes to increasing the level of automation (i.e. space constraints, hard to reach areas). Such processes usually require constant monitoring in order to prevent possible problems, and this task was usually carried out by human operators [2]. The application of machine vision appeared to be the logical step to take in order to assure quality and reliability to such operations and thanks to the constantly improving technology a great variety of apparatuses, capable of coping with almost every possible situation, are available on the market. An effective illumination of the working area is a crucial aspect when it comes to optimise the performance of such techniques. Unfortunately ideal light conditions are seldom easily achievable, for example if the vision system has to work within small areas, possibly close to surfaces with high reflectivity, such as metallic ones. In this case, locating and inspecting any feature on a component may become a particularly challenging task for the vision system. If the illumination within the working area is too weak, it might be hard even to locate all the features we are interested in, much less to inspect them. On the other hand, if the light condition is too bright, the reflection of the light 
towards the camera could over saturate the image and alter the edge profiles, therefore leading to measurement errors or even causing the vision system to miss some features completely. This work aims to investigate which factors mostly affect the accuracy in a typical feature recognition and measurement application, which consisted in inspecting a basic component to locate and measure a number of features on it. Three types of light were examined and for each one experiments were conducted using different settings and environmental conditions. The measurements were compared to those obtained by inspecting the component on a coordinate measuring machine (CMM), and the relative errors were calculated. Finally, a statistical analysis was carried out to assess the significance of the chosen factors.

\section{Methodology}

\subsection{Test apparatus}

The experimental apparatus consisted of a Perspex box containing the camera together with the light source and the test plate (Figure 1). The camera captured images of the sample plate at different distances and under varying light conditions, and sent them to the machine vision software described below for processing and inspection.

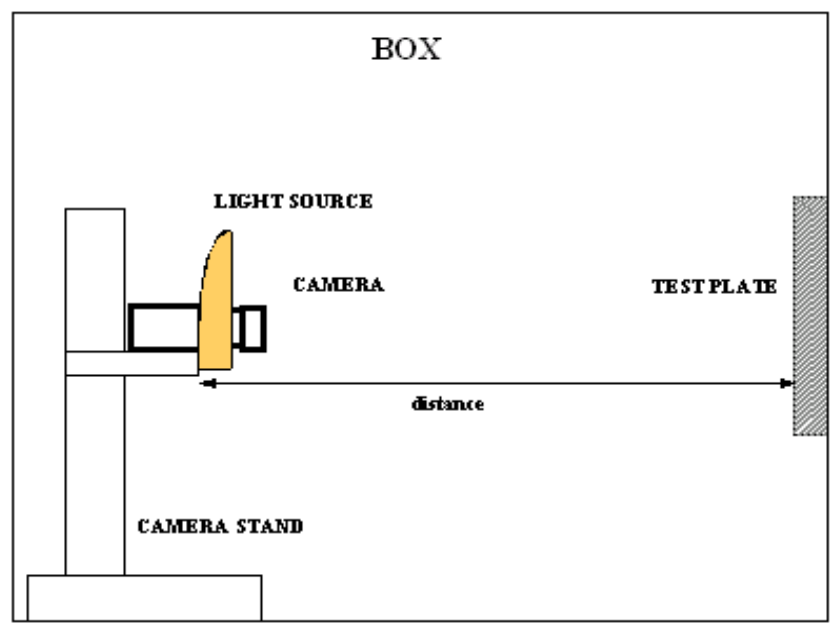

Figure 1 Experimental apparatus

The Perspex box, which simulates a working environment with significant space constraint, is shown in Figure 2. The two halves were coated with two coloured vinyl sheets in order to simulate different background conditions. The features of interest were machined on a plate made of Ti-6Al-4V (Figure 3), produced with different surface finish on the top and the bottom, which was bolted on an internal wall of the box. A Metris LK Evolution Series CMM, fitted with a Renishaw SP-25 probe with $2 \mathrm{~mm}$ diameter, was used to measure the features on the plate within an accuracy of $5 \mu \mathrm{m}$ prior to the vision experiments. 


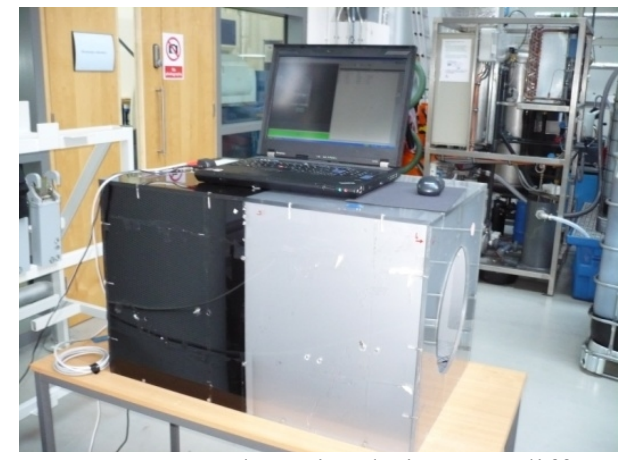

Figure 2 Perspex box simulating two different environments

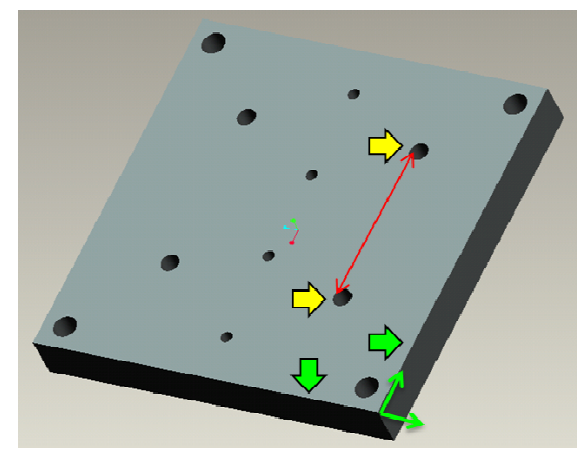

Figure 3 Test-piece

The images were captured by a Unibrain Fire-i 830b 2 Megapixel resolution camera, fitted with a linear polarizer and calibrated against a printed calibration grid with dots of known diameters and spacing. The Scorpion Vision Software 7.0 machine vision software from Tordivel was used for image inspection and feature measurement. The inspection aimed to identify the two edges on the bottom right corner of the plate (as indicated by the large green arrows in Figure 3) in order to set a datum reference frame on that corner. Subsequently, the machine vision software had to identify the two holes indicated by the yellow arrows, determine their positions with respect to the datum frame, and measure their radii and finally the distance between centres.

Three bespoke light sources were investigated in this work. Their performance in terms of light emission was assessed by estimating the average illuminance which they produced on the test surface, from three distances within a dark environment. All the measurements were carried out using a Testo 545 hand held light meter. The first light source (Figure 4a) uses the reflection from a dome shaped screen to reflect the light generated by a set of LEDs towards the object, thereby scattering the light beams and allowing a diffuse illumination of the surface. Because of this reason, this kind of devices is often called "cloudy day" light [3]. The device is equipped with two independent sets of LED which can be turned on alternatively or at the same time in order to vary the level of emittance.

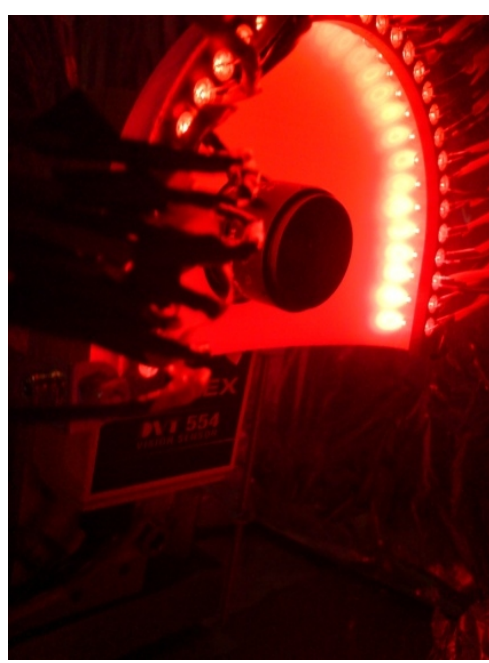

a: The Cloudy Day light

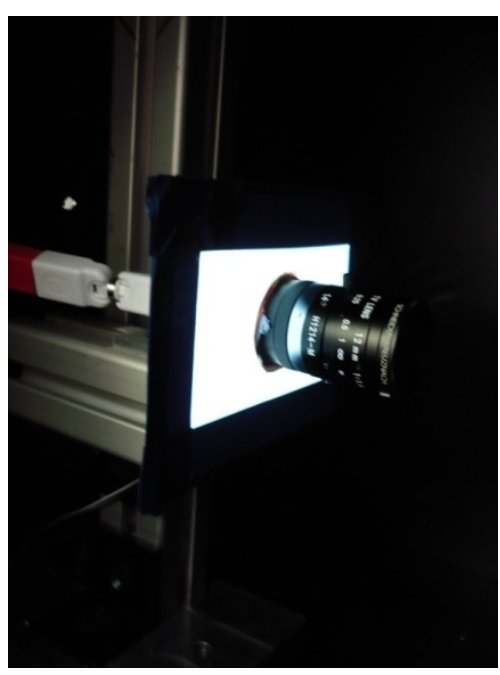

b: The ELS light

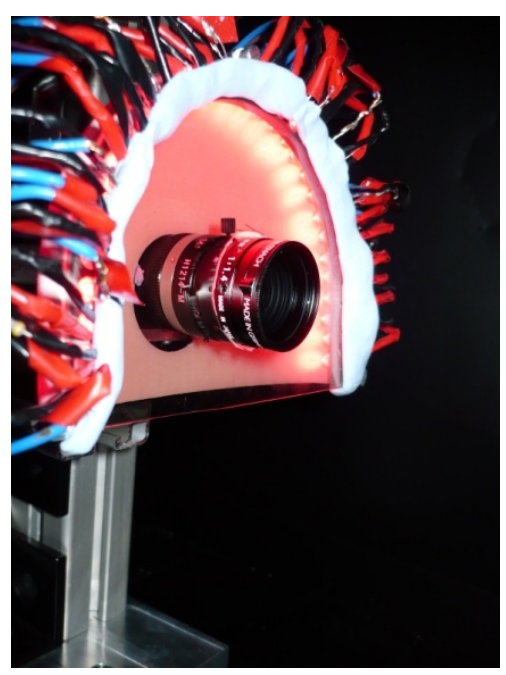

c: The Cloudy Day light fitted with polarising screen

Figure 4 Bespoke light sources 
The second type (Figure 4b) is an electroluminescent sheet which, although less powerful than an LED based light, is less cumbersome and can also be cut or bent to more convenient shapes. It is based on the electroluminescence phenomenon, i.e. the non-thermal generation of light as a result of the application of an electric field to certain substances [4]. The two levels of emittance examined during the experiments have been achieved by varying the emitting surface. Finally, the third type (Figure 4c) is a modified version of the cloudy day light, which was retrofitted with a polarizing screen in order to help minimising direct reflection towards the camera.

\subsection{Experimental procedure}

The problem of characterising and optimising the optical system was tackled using a design of experiments approach, DOE [5]. By means of this statistical method, the factors that are thought to have an effect on the system's outputs can be systematically studied. The relationship between the factors and their effects can be determined using a limited number of experiments. Thus, the main effects and their interactions can be calculated. A main effect is the effect of an independent variable averaging over all levels of the other independent variables in the experiment. An interaction between two factors is present when the effect of one factor depends on the level of the other factor.

In order to characterise the three different systems, an experiment was carried out. Six factors that are thought to have an effect on positional error and feature size were investigated: environment, distance to object, surface roughness, lens aperture, light level and shutter time. Table 1 provides the factors and their range for the experiments. The experimental design consisted on a fractional factorial with two replicates; blocking was conducted according to the two different environments. Three experimental matrixes (i.e. one for each system) consisting of 16 run were produced.

The settings for the experimental factors are reported in the following two tables.

Table 1 Experimental factors and their corresponding levels (* denotes LED system, **ELS and ***LED with polariser)

\begin{tabular}{|l|c|c|}
\hline \multirow{2}{*}{\multicolumn{1}{|c|}{ Factor }} & \multicolumn{2}{c|}{ Level } \\
\cline { 2 - 3 } & Low & High \\
\hline Environment illuminance [1x] & 0 & 19 \\
\hline Distance to object $[\mathrm{mm}]$ & 200 & 300 \\
\hline Surface roughness $[\mu \mathrm{m}]$ & 0.37 & 1.24 \\
\hline Lens aperture [mm] & $\mathbf{f} / 1.4$ & $\mathbf{f} / 4$ \\
\hline Shutter values & 1400 & 1600 \\
(Shutter times) & $(40.5 \mathrm{~ms})^{*}$ & $(60.5 \mathrm{~ms})^{*}$ \\
& 2400 & 2600 \\
& $(780 \mathrm{~ms})^{* *}$ & $(2780 \mathrm{~ms})^{* *}$ \\
& 2300 & 2500 \\
& $(671 \mathrm{~ms})^{* * *}$ & $(1780 \mathrm{~ms})^{* * *}$ \\
\hline
\end{tabular}

Table 1 reports both shutter values and the equivalent shutter times, calculated according to the equations reported in the camera user manual [6].

Table 2 describes the sixth factor, i.e. the light level, through a characterization of the three light sources used during the experiments. The minimum and the maximum level of light are defined in terms of the average illuminance produced on the test surface from three different distances. 
Trends in Aerospace Manufacturing 2009 International Conference

IOP Publishing

IOP Conf. Series: Materials Science and Engineering 26 (2011) 012019

doi:10.1088/1757-899X/26/1/012019

Table 2 Determination of the light level through a characterization of the light sources used during the experiments $(*$ denotes LED system, **ELS and ***LED with polariser)

\begin{tabular}{|c|c|c|c|c|c|c|}
\hline & \multicolumn{6}{|c|}{ Measured illuminance on the test surface } \\
\hline \multirow{2}{*}{$\begin{array}{c}\text { Distance } \\
(\mathrm{mm})\end{array}$} & \multicolumn{2}{|c|}{$\underset{*}{\text { Illuminance }}(\mathrm{lx})$} & \multicolumn{2}{|c|}{$\underset{* *}{\text { Illuminance }}(\mathrm{lx})$} & \multicolumn{2}{|c|}{$\underset{* * *}{\text { Illuminance }}(\mathrm{lx})$} \\
\hline & $\min$ & Max & $\min$ & Max & $\min$ & Max \\
\hline 200 & 22 & 44 & 4 & 11 & 6 & 12 \\
\hline 250 & 12 & 24 & 3 & 7 & 3 & 6 \\
\hline 300 & 8 & 16 & 2 & 5 & 2 & 4 \\
\hline
\end{tabular}

Once the key factors were identified, a second set of tests was carried out in order to optimise them and to provide useful guidelines on how to minimise measurement errors in different scenarios. A second fractional factorial design of experiments was performed. The factors investigated were the environment illuminance, shutter time, distance to object and surface roughness. Two replicates were conducted leading to 16 experimental runs.

\section{Results and analysis}

\subsection{Characterisation of cloudy day light}

The results obtained after testing the cloudy day light showed that shutter and environment illuminance had the strongest influence on the mean relative error in all the measured features: position of the holes, radii and distance between centres. Figure 5 produces a plot of marginal means (difference between the average of the output corresponding to the low parameter value and the one corresponding to the high parameter value. The more significant the effect of the parameter, the bigger the difference between these two averages, illustrated by the plots gradient) for the $\mathrm{x}$ position of the centre of the holes, but these considerations can be definitely extended to the other features (i.e. y position, hole size and distance between centres).

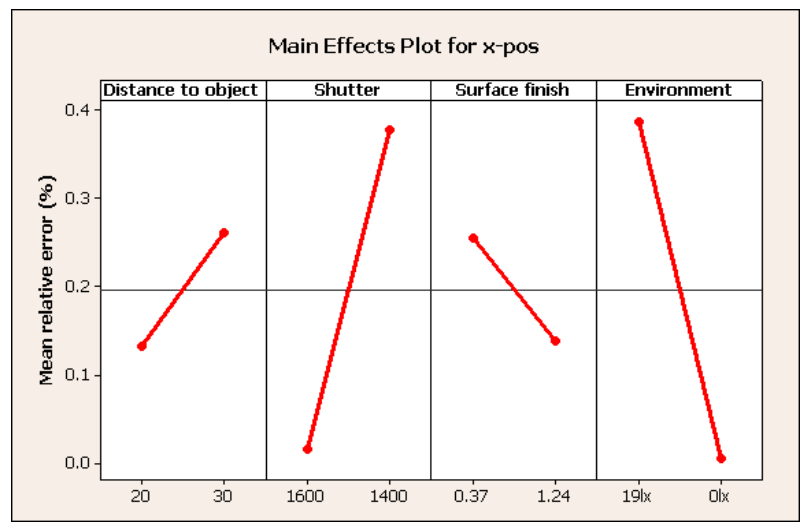

Figure 5 Main effect plot for x-position

In particular the relative error was low for high shutter values and low amount of illuminance on the working environment, with the relative error dropping from $100 \%$ (which means that the machine vision software could not identify the vertical and horizontal edges, thereby being unable to set a datum frame in order to locate the holes) to $0.34 \%$ when the surface finish is $0.37 \mu \mathrm{m}$ and from $2.10 \%$ to $0.72 \%$ when it is $1.24 \mu \mathrm{m}$, which constitutes an ideal condition for enhancing the contrast between the test-piece surface and the background. The experiments highlighted that the relative error in size, 
i.e. the diameter of the holes, was a lot larger than the positional error (up to $9.16 \%$ with the lowest surface finish), assuming that it was possible to set the datum frame. This large difference is explained by the fact that the CMM inspected the holes at $1.5 \mathrm{~mm}$ below the surface in order to prevent damaging the probe, whilst the machine vision software obviously evaluated the contour of the holes on the top surface, where surface finish, direct reflection and slight imperfections due to machining process may alter the contour slightly. Distance from the object and surface roughness, as separate factors, had a relatively modest influence on the relative error. However, a strong interaction was identified between these two factors, as shown in Figure 6.

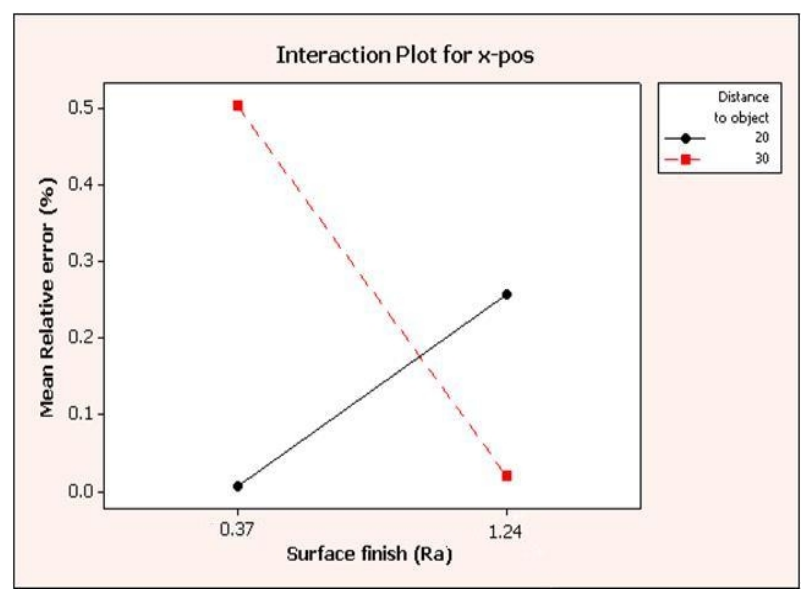

Figure 6 Interaction plot for x-position

Specifically, for smoother surfaces the mean relative error decreases with distance to object; on the other hand, for rougher surfaces the error increases with distance to object. It appears that surface asperities can influence the reflection towards the camera by generating random bright spot on the test surface [7]. Their influence can be reduced by carefully positioning the light source but it cannot be completely eliminated and this can require a great deal of image processing from the machine vision software in order to minimise measurement errors.

\subsection{Characterisation of electroluminescent sheet light}

Experiments on the ELS panel proved that the environment had a significant effect on the relative error for position, size and distance between centres (Figure 7).

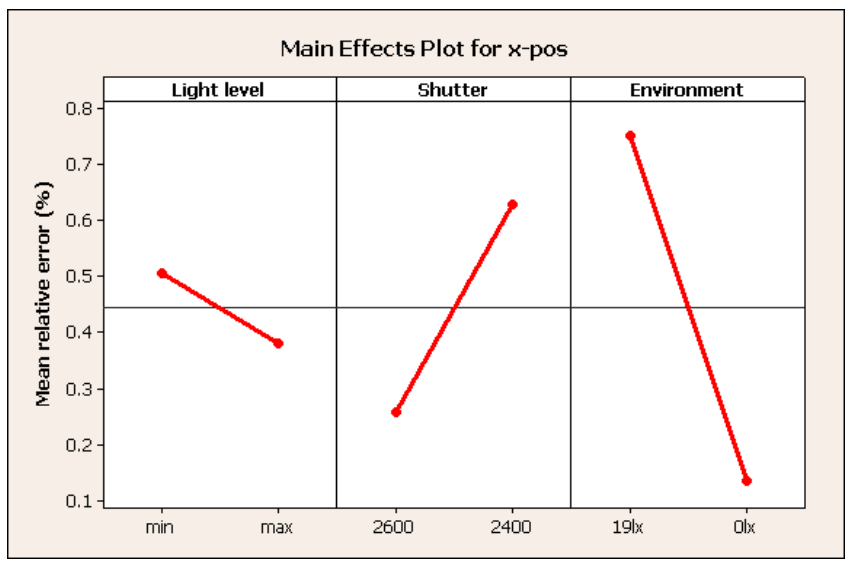

Figure 7 Main effect plot for x position error 
Like the previous case, the error is lower for the dark background and higher values of shutter time led to a reduction of the error. Besides the consideration about the role of the asperities, it should be noted that, in this particular application, having a bright background may cause the machine vision software to interpret the shadows deriving from the thickness of the plate as false edges (see Figure 8), therefore increasing the possibility of measurement errors. For high shutter values, the camera was completely blinded by the light reflection, thereby making impossible to locate features at all and producing a $100 \%$ error as a result.

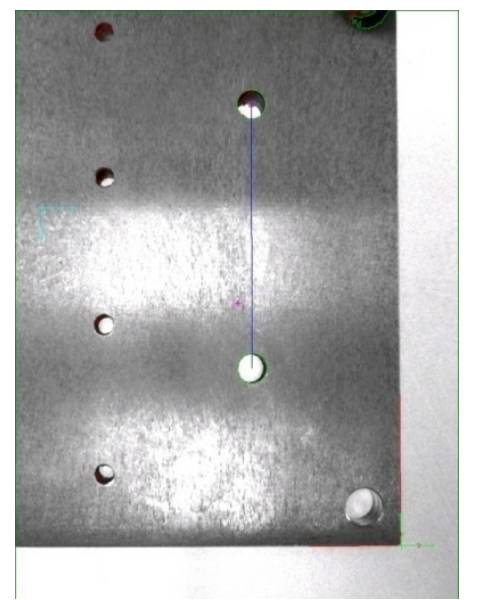

Figure 8 The bright background can generate shadows which might be interpreted as edges by the machine vision software.

The statistical analysis according to the fractional factorial design pointed out that there was an interaction between the light level and both the shutter and the background. Minimum light level was not strong enough to affect the error when the shutter changed, possibly because the shutter range is not wide enough for the machine vision software to appreciate the difference. On the other hand, maximum light level affects the output when the shutter changes, presenting the lowest relative error when high shutter values are used.

Although shutter and background have a large influence on the relative error as separate factors, they do not appear to affect each other. The relative error for the size was once again larger than those measured for the other features, due to the difference between the two methods of measurement adopted (machine vision and CMM).

\subsection{Characterisation of modified cloudy day light}

Adding a polarizing screen to the cloudy day light enabled a large amount of direct reflection to be eliminated provided that the polarizer mounted on the top of the objective lens is conveniently adjusted. As a result, a more homogeneous distribution of the amount of light is achieved on the test surface, although the average illuminance produced by the light is lower than that generated by the standard cloudy day light.

Similar to what has been found after testing the standard version of this light source, results show that shutter and environment have a strong effect on positional, size and distance error. Figure 9 reports the plot of the marginal means for the error in size, but these considerations can be extended to the other features as well. As for the error in size, the same considerations about how the features were measured by both the machine vision software and CMM apply. The difference between the relative 
error for the size and that for the other features should therefore be not surprising. It has been found that the relative error decreased with higher shutter values and dark environment.

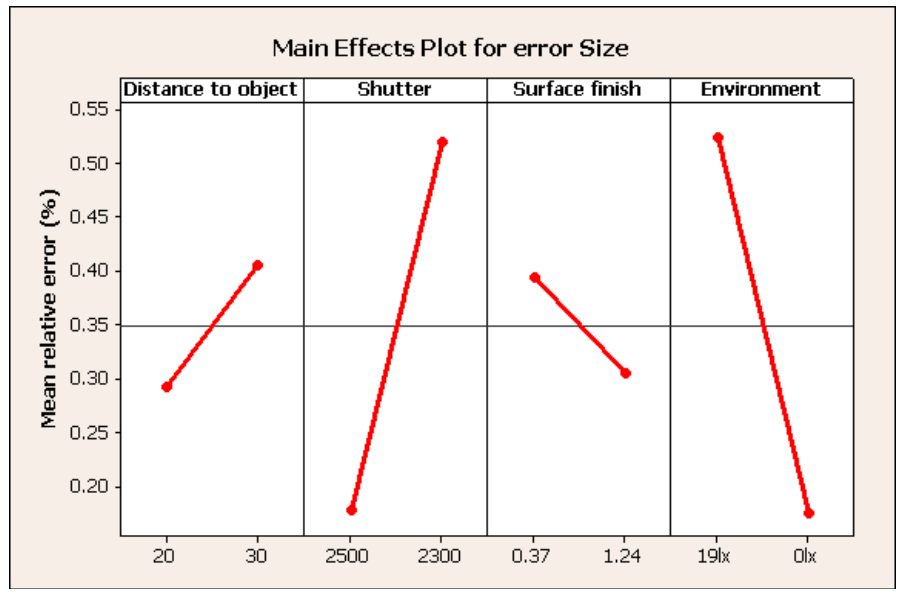

Figure 9 Main effects plot for error in size

In particular the relative error was low for high shutter values and low amount of illuminance coming from the background (from $9.03 \%$ to $2.14 \%$ depending on surface finish and distance to object) which constitutes an ideal condition for enhancing the contrast between the test surface and the background.

Distance from object and surface roughness affect the relative error as well, although their influence is considerably lower compared to that of the background and shutter. However, a strong interaction was identified between these two factors. The interaction between distance to object and surface finish had a statistically significant effect on positional, size and distance error. Specifically, for high distances from the object large values of Ra caused the relative error to be very low. The same result is found when low Ra values and small distances are considered. As for the influence of Ra, it appears that, even in this case, surface asperities influenced the reflection towards the camera. A careful choice and positioning of the light source can reduce this problem greatly [5] but it cannot eliminate it completely. This can require a great deal of image processing from the machine vision software in order to minimise measurement errors.

\section{Optimisation of the key factors: results and analysis}

The results from the previous section have shown that four factors proved to have significant influence on the relative error: environment illuminance, shutter, surface roughness and distance from the object.

Based on the results, a second set of tests has been carried out in order to optimize these factors and to provide useful guidelines on how to minimize measurement errors in different scenarios, according to the settings reported in Table 3.

Table 3 Experimental factors and their corresponding levels for the second set of experiments

\begin{tabular}{|l|c|c|}
\hline \multirow{2}{*}{ Factor } & \multicolumn{2}{c|}{ Level } \\
\cline { 2 - 3 } & Low & High \\
\hline Environment illuminance $[\mathrm{lx}]$ & 0 & 19 \\
\hline Distance to object $[\mathrm{mm}]$ & 200 & 250 \\
\hline Surface roughness $[\mu \mathrm{m}]$ & 0.37 & 1.24 \\
\hline Shutter & 1800 & 2100 \\
& $(171 \mathrm{~ms})$ & $(471 \mathrm{~ms})$ \\
\hline
\end{tabular}


The experiments were carried out using the modified cloudy day light and showed that all of the four factors had a significant effect on positional error. Figure 10 plots out the marginal means for the horizontal position of the centres of the holes but the same considerations can be extended to the other features as well. High shutter and low distance to object values caused the relative error to decrease.

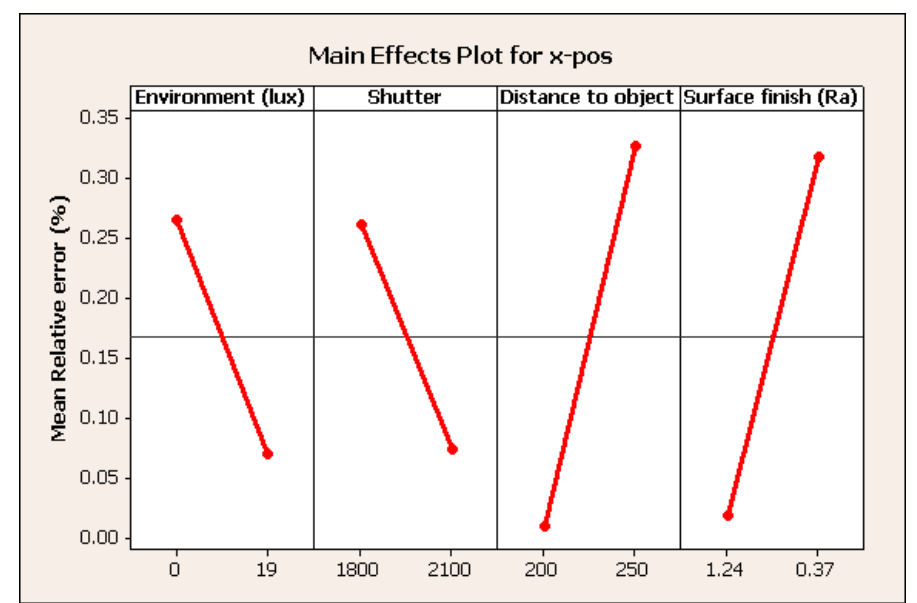

Figure 10 Marginal means for x-position. Main effects plot.

This time, the error was lower with the bright background (the lowest relative error was $0.24 \%$, against $0.86 \%$ within the dark environment), suggesting that the contrast between the plate and the background was high enough for the machine vision software to clearly locate the edges despite the fact that the shutter values were generally lower than the previous cases.

Figure 11 reports the interactions that occurred among the key factors which influenced the relative error, according to the statistical analysis.

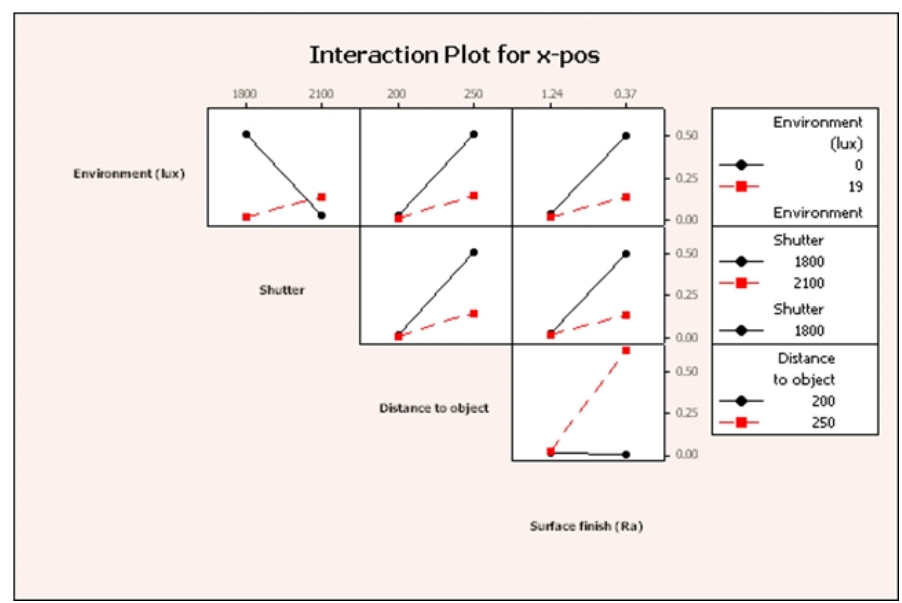

Figure 11 Interaction plot for the key factors, $\mathrm{x}$ position

It appears that background and shutter had the strongest interaction. In particular with a bright background low values of the shutter should be used in order to minimise saturation of the area of interest. On the other hand, in dark environment high shutter values should be preferred in order to maximise the contrast between the edges of the plate and the surrounding area.

Low interaction occurred between environment and distance to object, and between environment and surface finish. Whether the background was dark or bright the estimated error decreased with low distance and high Ra. Interaction occurred also between distance from object and surface finish, 
according to the observations that have been made previously concerning the role that surface asperities play on direct reflection.

The analysis on size error highlighted that the key factors were influential in the same way they affected the positional error, as shown in Figure 12 (the lowest relative error was $1.38 \%$, against $2.01 \%$ within the dark environment). Only this time the incidence of environment appeared to be slightly heavier.

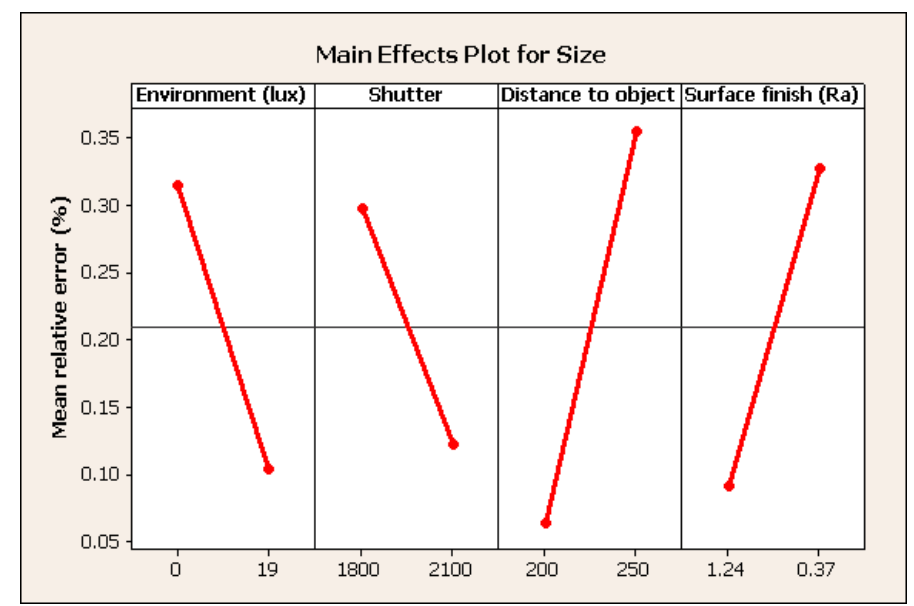

Figure 12 Main effects plot for error in size - Marginal means

The size error was lower for the bright environment and decreased with decreasing distance to object. High values of shutter and surface roughness lead to minimisation of the error, too.

The interactions between the factors had a significant effect on size error (Figure 13). Background illuminance and shutter time had the strongest interaction again. In particular with a bright background low shutter values should be preferred in order to minimize the relative error.

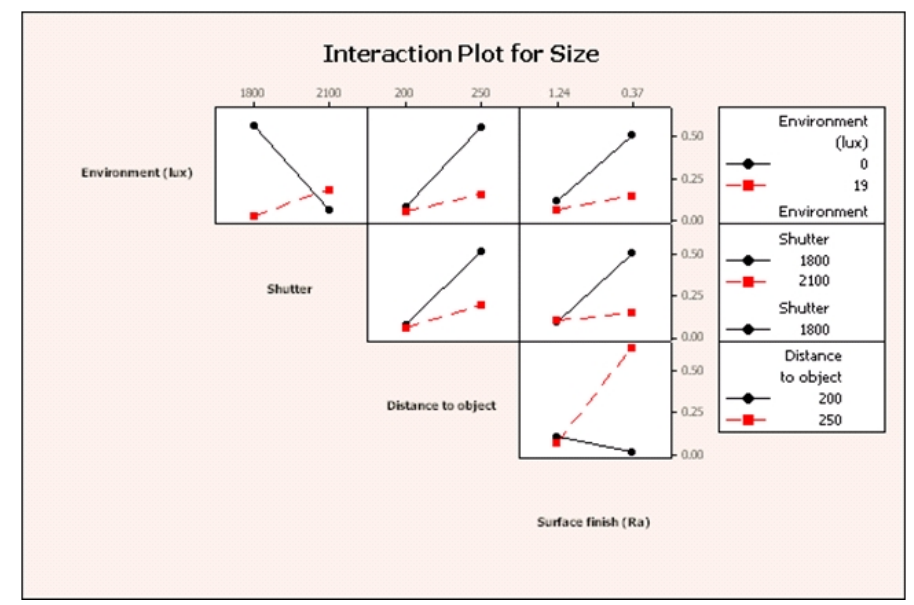

Figure 13 Interaction plot for error in size

Low interaction occurred between environment and distance to object, and between environment and surface finish. Whether the background was dark or bright the estimated error decreased with low distance and high surface roughness. Interaction occurred also between distance from object and surface finish (see section 3.1 regarding the observation about the role of the surface asperities in light reflection). 
The four key factors also affected the relative error of the distance between centres (the lowest relative error being $0.03 \%$ within bright environment and $0.3 \%$ within dark environment), but this time the influence of the distance to object and the surface finish, as separate factors, was a lot larger than the influence from background and shutter (Figure 14).

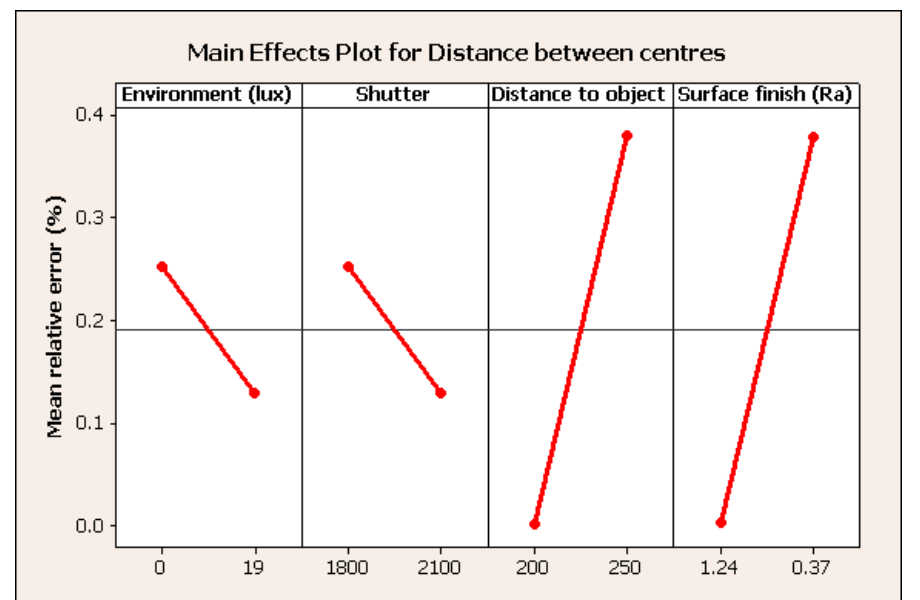

Figure 14 Main effects plot for error in distance between centres - Marginal means

Environment and shutter had a strong interaction with each other in the same way as shown regarding the other features, suggesting using high shutter values in order to lower the error when the background is dark and vice versa.

\section{Conclusions and future work}

The experiments on the different light sources highlighted that four factors played a key role, among those which have been considered in the experimental design. These factors are environment illuminance, shutter time, surface roughness and distance to object and they had an influence on the relative error as independent factors, but their interactions proved to affect the error as well.

The optimisation of the four key factors proved that shutter time and environment were the most significant ones in the majority of the situations. The experiments indicated that interaction between shutter time and environment produced the best results by using lower shutter values with bright backgrounds (ideally around 1800 UNITS) and vice versa, higher shutter values with dark back grounds (ideally above 1900 UNITS).

Interactions of surface roughness with both the environment and the shutter were also important: high values of these two factors combined with smoother surfaces often produced direct reflection which disturbed the identification of the features of interest.

Not surprisingly, the measured error was smaller for short distances, and its increment with the distance was more evident if a darker environment and/or a smaller shutter were involved.

The measured error values, sometimes above $2 \%$, indicated that there is still room for improvement in both the set up and the experimental procedure. In future experiments, higher values of shutters are definitely to be explored since combining the polarizer fitted on the camera and the one fitted on the light source enables the direct reflection to be considerably reduced. Camera calibration procedure could be improved by producing a more accurate calibration grid and also making the light condition outside the box more stable.

Again a weak interaction was noted between the environment and the other factors, and between the distance to object and the surface finish. 
Whether the background was dark or bright the estimated error decreased with low distance and high surface roughness and the same result has been found when the shutter is considered.

Interaction occurred also between distance to object and surface finish, according to the considerations that have been made in section 3.1, regarding the effect of surface asperities on light reflection toward the camera. Their influence could be reduced by carefully positioning the light source but it cannot be eliminated entirely and this can require image processing from the machine vision software so that measurement errors can be minimised.

\section{Acknowledgements}

The authors acknowledge the support of the European Commission through the 6th Framework Programme under Priority 3 NMP Call FP6-2004-NMP-NI-4 (AFFIX; contract number 026670)

\section{References}

1. Perks, A, "Advanced vision guided robotics provide "future-proof" flexible automation", Assembly Automation, 26 (3) (2006), 216-220, Emerald Group Publishing Limited;

2. Golnabi, H, Asadpour, A, "Design and application of industrial machine vision systems", Robotics and Computer Integrated Manufacturing, 23 (2007), 630-637, Elsevier;

3. Braggins, D, “Illumination for machine vision", Sensor Review, 20 (1) (2000), 20-23, Emerald Group Publishing Limited;

4. Ono, Y. A, “Electroluminescent displays”, (1995), World Scientific Publishing Co. Pte. Ltd, Singapore;

5. Montgomery, D.C., “Design and analysis of experiments”, (2000) 643, Wiley, Chichester;

6. Unibrain, “Fire-I 530/630/830 User Operation Manual”, (2008), http://www.unibrain.com/download/pdfs/ub1394cam/ubultracompact manual_v1.pdf;

7. Kurada, S, Bradley, C, “A review of machine vision sensors for tool condition monitoring”, Computers in Industry, 34 (1997), 55-72, Elsevier. 